\title{
Histologic Features and Differential Diagnosis of Endometrial Polyps; An Update and Review
}

\author{
Ali Dastranj Tabrizi*
}

\begin{abstract}
Among the endometrial lesions, endometrial polyps (EPs) are commonly seen in the premenopausal and postmenpuasal age group and are found in $25 \%$ of endometrial specimens performed for abnormal uterine bleeding. EPs are generally considered as local hyperplasitic lesions of endometrial stroma which covered by epithelium. These lesions are seen single or multiple, sessile or pedunculated and the size range differs from millimetres to centimetres. In $10 \%$ of cases they mimic endocervical polyp due to prolapsus from external os. In a case of sufficient clinical, hysteroscopic and imaging information, the histologic diagnosis is usually straightforward. From clinical and prognostic standpoint, it should be emphasized that rarely these lesion may harbour premalignant and malignant lesions particularly in older age group. Therefore careful histologic examination for rule out of raised malignant lesions should be considered. In the other hand in rare cases these lesions may show metastatic involvement particularly from lobular breast carcinoma. Prevalence of these lesions in association with clinical presentation and histologic features will be presented and differential diagnosis with special emphasis on rule out of malignant lesions will be discussed.

Keywords: Endometrial, Polyps, Review
\end{abstract}

\section{Introduction}

Among the endometrial lesions, endometrial polyps (EPs) are commonly seen in the premenopausal and postmenpuasal age group and are found in $25 \%$ of endometrial specimens performed for abnormal uterine bleeding (1). EPs are generally considered as local hyperplasitic lesions of endometrial stroma which covered by epithelium. These lesions are seen single or multiple, sessile or pedunculated and the size range differs from millimetres to centimetres. In $10 \%$ of cases they mimic endocervical polyp due to prolapsus from external os $(2,3)$. In a case of sufficient clinical, hysteroscopic and imaging information, the histologic diagnosis is usually straightforward. From clinical and prognostic standpoint, it should be emphasized that rarely these lesion may harbour premalignant and malignant lesions particularly in older age group. Therefore careful histologic examination for rule out of raised malignant lesions should be considered. In the other hand in rare cases these lesions may show metastatic involvement particularly from lobular breast carcinoma. Prevalence of these lesions in association with clinical presentation and histologic features will be presented and differential diagnosis with special emphasis on rule out of malignant lesions will be discussed.

\section{Prevalence}

In the 2402 office hysteroscopy performed on women with mean age of 39.4 years old for several indications, the prevalence of EP was $27.2 \%$ (4). Based on used diagnostic method and study group the mentioned rate varies between $6 \%$ to $32 \%$ and this rate increases with age (5-7). In another large study carried out on 686 Danish women in range of 20-79 years of old who accepted to be examined by both transvaginal sonography and saline contrast sonohysterography, the prevalence of EP was $7.8 \%$ (5). In this study the EPs were rare $(0.9 \%)$ between young women and in women older than 30 years the prevalence was 9.2\%. EPs are rarely found in OCP user whereas in the women who were on hormone replacement therapy the prevalence was much higher (2.1\% vs. $25 \%)$ (5). Respect to hormones as an etiologic factor, high frequency of endometrial hyperplasia was found in the non-polyploid region of endometrium in premenopausal and postmenopausal women (8).

\section{Clinical Presentation}

The usual clinical presentations of EPs may include menorrhagia, intermenstrual bleeding, postmenopausal bleeding and infertility. However the majority of patients may be asymptomatic and the prevalence could be similar in symptomatic and asymptomatic women (5). These findings indicates that there is no consistent relationship between abnormal uterine bleeding and existence of EPs and why hysteroscopic resection of EPs does not always result in relief of symptoms (9).

A pathologist should be alert that in the most cases of protruded EPs, the usual clinical diagnosis will be endocervical polyp by the gynaecologist. Regard to tamoxifen, EPs are the most common pathology that could be seen in these patients. In these patients the prevalence of

Received 9 April 2016, Accepted 17 August 2016, Available online 15 September 2016

Women's Reproductive Health Research Center, Tabriz University of Medical Sciences, Tabriz, Iran.

*Corresponding Author: Ali Dastranj Tabrizi, MD; Email: dastranjt@gmail.com 
EPs which discovered by hysteroscopy and confirmed by histology was $38.6 \%$ and $30.9 \%$ respectively (10). In another study the presence of EPs was recorded in $54.6 \%$ of postmenopausal patients who were on tamoxifen (11). It seems that tamoxifen related polyps have a tendency to be larger and commonly presented as multiple lesions.

\section{Histologic Features}

Histologically, EPs are considered raised lesions composed of mostly stromal proliferation in association with various glandular alteration covered by epithelium. The range of glandular change differ from cystically dilated atrophic glands through hyperplastic glands to rare endometrial carcinoma (Figure 1A). Frequently, the proliferated glands represents as disordered proliferative glands in a compact or fibrotic stroma. In fact the latter feature is characteristic for EP and more often seen in the polyps due to tamoxifen therapy. In practice any raised endometrial lesion with the mentioned histologic pattern that lined by epithelium in three sides should be considered EP. However the polyploid appearance of endometrium treated with high dose progesterone should be considered in differential diagnosis. In this case decidualized endometrial stroma in association with inactive and exhausted glands usually leads to correct diagnosis. Another useful diagnostic feature is presence of glands which their longer axis are in parallel with the surface epithelium (Figure 1B). The glands may be inactive and dilated but functional glands could be seen in the polyps also. Existence of thick walled and hyalinized vessels often considered as a useful histologic feature and some investigators insists that these vessels should be located near the surface epithelium (Figure 1C).

Although it is rare occurrence, both endometrioid and serous carcinoma in association with their precursor lesions may involve or originate from the EPs. In this context it is essential to examine any atypical foci with especial care.
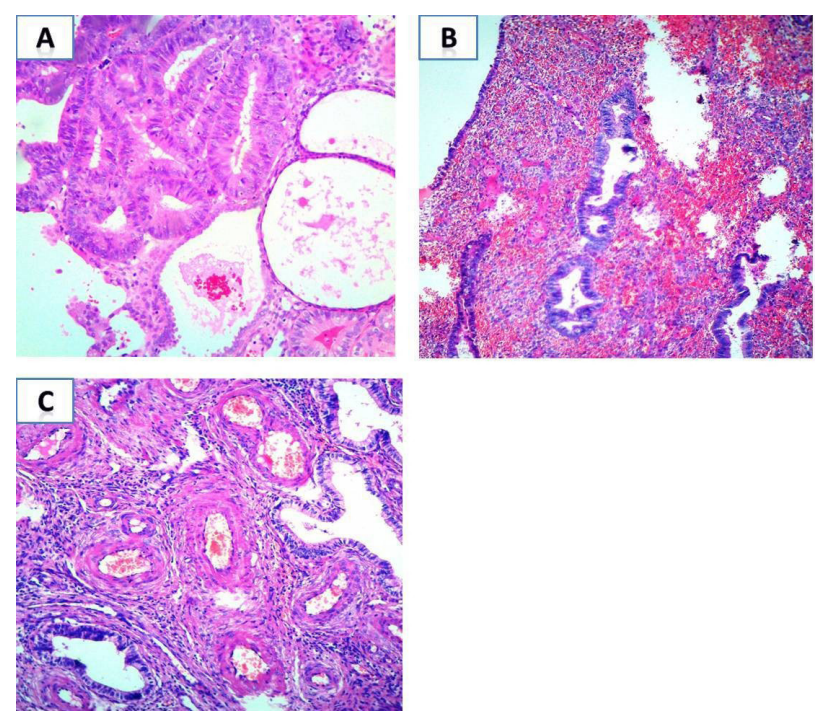

Figure 1. (Hematoxylene \& Eosine) (A) Atypical hyperplasia partially involved an endometrial polyp (200X). (B) Note the long axis of glands in parallel with surface epithelium (100X). (C) Numerous thick walled vessels in the EP (100X).
In some cases the nuclear atypia is often subtle and may be easily overlooked. Although the assessment of nuclear atypia in the endometrial glands is very subjective and may be problematic, however presence of round and vesicular nuclei with loss of polarity should be considered as a worrisome finding (12). Indeed, rather than obvious nuclear atypia, presence of different nuclear features between normal and atypical glands should be considered one of the characteristic findings of endometrial intraepithelial neoplasia (EIN) raised from EP. In fact existence of distinctive glands crowding in association with comparable nuclear feature between normal and crowded area may be used for detection of EIN in a polyp, although other corresponding findings of EIN should be found. In rare cases, endometrial intraepithelial carcinoma (EIC) - that currently considered as a minimal invasive serous carcinoma rather than its precursor - may be found in the EPs or originate from these lesions. Finding of any marked nuclear atypia with prominent nucleoli and brisk mitotic activity should be considered highly suspicious particularly in the superficial epithelium. In this setting high mitotic activity in association with apoptotic bodies are constant features of serous carcinoma and can be confirmed by immunostaining for p53, ki67 and p16 (13) (Figure 2).

Distinction of benign EP from uterine adenosarcoma may represent a diagnostic challenge. Most of the uterine adenosarcomas are large polyploid masses that usually protruding from cervical os. Histologically presence of any of the following features; marked stromal hypercellularity (including periglandular cuffing), stromal nuclear atypia and mitotic figures $\geq 2 / 10 \mathrm{HPF}$ in the stromal cells warrants a diagnosis of adenosarcoma. In this setting, partial involvement of the polyps with some of these features may be interpreted as atypical EP (14).

EPs with atypical (bizarre) stromal cells may also represents a potential diagnostic pitfall. These cases usually

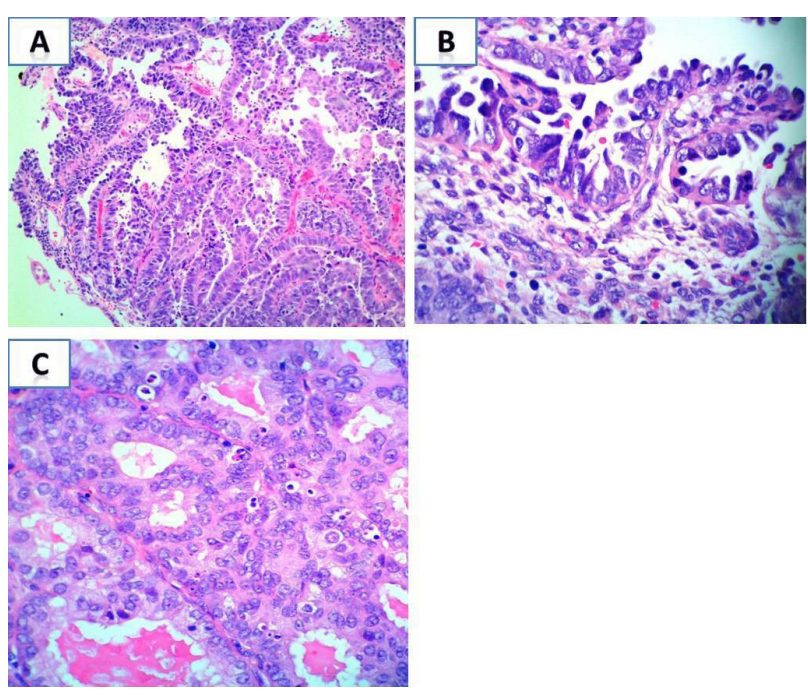

Figure 2. (Hematoxylene \& Eosine) (A) Endometrial serous carcinoma in an endometrial polyp (100X). (B) Endometrial intraepithelial carcinoma (EIC) is seen in the surface epithelium (200X). (C) An EP has been involved by endometrial intraepithelial neoplasia (EIN) (400X). 
are accidental findings, however presence of cells with large, hyperchromatic, multilobulated nuclei or multinucleated stromal cells which can be distributed in focal or multimodal pattern, may be interpreted as a worrisome finding. Smudged pattern of nuclear chromatin similar to atypical leiomyoma, lack of periglandular cuffing, absence of mitotic activity and very low proliferative index in these cells which can be confirmed by very low immunoreactivity to ki67 are useful findings for rule out of malignancy $(15,16)$ (Figure 3).

\section{Discussion}

EPs are benign localized glandular and stromal overgrowth that represent as sessile or pedunculated lesion, may be seen single or multiple and varies in size from millimetres to centimetre $(2,3,17)$. Based on population study, its incidence varies between $7.8 \%$ to $34.9 \%(18,19)$. It is estimated that this prevalence may be higher in infertile women. In a prospective study of 1000 infertile women who underwent hysteroscopy before in vitro fertilization, it was found that $32 \%$ of patients had EP (20).

From mechanistic perspective, it seems that endometrial stimulation by estrogen, genetic mutations, age, obesity, hypertension and diabetes are implicated in pathogens of EPs (21-25). It worth note that age may be the most important predisposing factor. It is well known that patients who use tamoxifen are in higher risk and the prevalence of EP in these patients may raise up to $32 \%$ (26). .Although these polyps are generally considered benign lesions, in rare cases they may harbour premalignant, malignant and evenly metastatic lesions. In a study (unpublished data) we found 26 malignant EPs over 20 years that involved by serous carcinoma.

From clinical perspective, the EPs usually discovered by imagining or hysteroscopic evaluation of the patients who referred by abnormal uterine bleeding or infertility. However these lesions may be protruded from cervix and presented as endocervical polyp or protruded myoma. In the later circumstances, careful examination of the lesion for rule out of endometrial or cervical adenosarcoma is necessary. In the same way scrutinized evaluation of glandular epithelium including surface epithelium to finding of any true nuclear pleomorphism and prominent nucleoli should be kept in mind for rule out of minimal endometrial serous carcinoma. In addition it should be emphasized that precursors of type I endometrial carcinoma including EIN or complex atypical hyperplasia may be found in the EPs. These premalignant or malignant lesions may be confined to the polyps or can be seen in other regions of endometrium. Therefore it is reasonable to examine carefully the rest of endometrium in hysterectomy specimens harbouring EPs.

EPs may be involved by metastatic lesions also. Although endometrial metastatic involvement is unusual and less common than vaginal and ovarian involvement, it is typically occurring secondary to breast lobular carcinoma (27-29). Hence recognition of this occurrence in breast cancer patients who presented with abnormal uterine
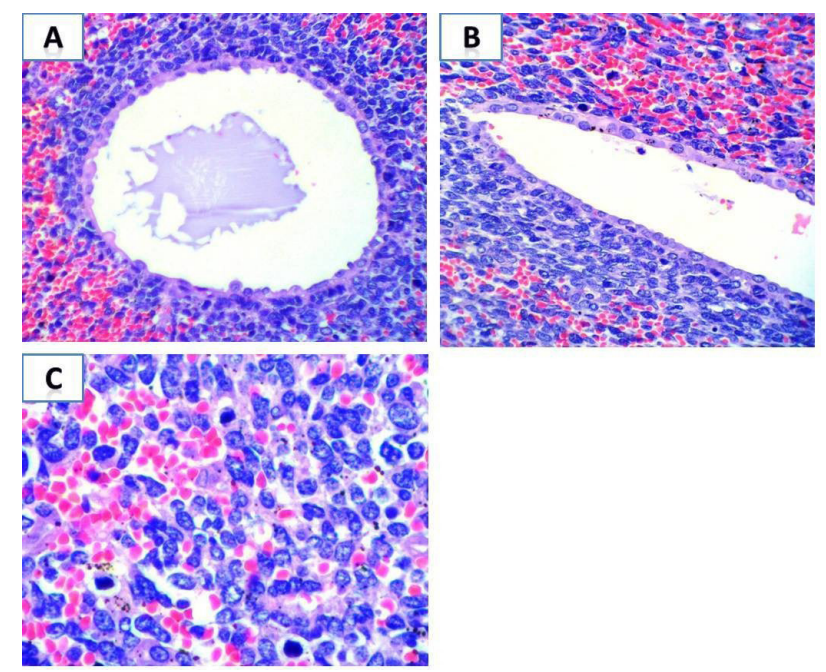

Figure 3. (Hematoxylene \& Eosine) (A) obvious periglandular cuffing in uterine adenosarcoma (200X). (B) Numerous mitotic activity in the stromal component of adenosarcoma (200X). (C) An adenosarcoma with prominent nuclear atypia in the sarcomatous component (400X).

bleeding should promptly indicate for careful examination of endometrium in these patients.

Regards to other types of endometrial carcinoma, it is worth to note that clear cell and mucinous carcinoma can be found in the EPs also (30). In the minimal endometrial serous carcinoma or EIC, the common genetic mutation which can be confirmed with IHC method is P53 mutation (31). Therefore in the suspicious polyps, IHC staining for P53 will be useful in confirmation of diagnosis. As in other types of gynaecological lesions (32), IHC staining for Ki67 can be applied for confirmation of increased cellular proliferation in the EPs. Hence Ki67 may be used as a useful marker in recognition of malignant or premalignant lesions in the EPs.

\section{Conclusion}

Although EPs as relatively common endometrial lesions (particularly in patients with abnormal uterine bleeding and infertility) are considered benign lesions, a range of atypical, premalignant and malignant changes can be found in both glandular and stromal components of these lesions. Therefore careful attempt in microscopic examination of these lesions should be accomplished.

\section{Ethical Issues}

We have no ethical issues to declare.

\section{Conflict of Interests}

We have no conflict of interest to declare.

\section{Financial Support}

None.

\section{Acknowledgments}

None. 


\section{References}

1. Clement PB, Young R. Atlas of Gynecological Surgical Pathology. 3rd ed. St. Louis Saundersher: Elsevier; 2014.

2. Elias RT, Pereira N, Karipcin FS, Rosenwaks Z, Spandorfer SD. Impact of newly diagnosed endometrial polyps during controlled ovarian hyperstimulation on in vitro fertilization outcomes. J Minim Invasive Gynecol 2015;22:590-4. doi: 10.1016/j.jmig.2014.12.170.

3. Rackow BW, Jorgensen E, Taylor HS. Endometrial polyps affect uterine receptivity. Fertil Steril. 2011;95: 2690-2. doi: 10.1016/j.fertnstert.2010.12.034.

4. Capmas P, Pourcelot AG, Giral E, Fedida D, Fernandez H. Office hysteroscopy: A report of 2402 cases. J Gynecol Obstet Biol Reprod (Paris). 2016;45: 445-50. doi: 10.1016/j.jgyn.2016.02.007.

5. Dreisler E, Stampe Sorensen S, Ibsen PH, Lose G. Prevalence of endometrial polyps and abnormal uterine bleeding in a Danish population aged 20-74 years. Ultrasound Obstet Gynecol. 2009;33:102-8. doi: 10.1002/uog.6259.

6. Nagele F, O’Connor H, Davies A, Badawy A, Mohamed H, Magos A. 2500 Outpatient diagnostic hysteroscopies. Obstet Gynecol. 1996;88:87-92. doi: 10.1016/0029-7844(96)00108-1.

7. Vilodre LC, Bertat R, Petters R, Reis FM. Cervical polyp as risk factor for hysteroscopically diagnosed endometrial polyps. Gynecol Obstet Invest. 1997;44: 191-5.

8. Rahimi S, Marani C, Renzi C, Natale ME, Giovannini P, Zeloni R. Endometrial polyps and the risk of atypical hyperplasia on biopsies of unremarkable endometrium: a study on 694 patients with benign endometrial polyps. Int J Gynecol Pathol. 2009;28:522-8. doi: 10.1097/PGP.0b013e3181a42228.

9. Henriquez DD, van Dongen $\mathrm{H}$, Wolterbeek $\mathrm{R}$, Jansen FW. Polypectomy in premenopausal women with abnormal uterine bleeding: effectiveness of hysteroscopic removal. J Minim Invasive Gynecol. 2007;14: 59-63. doi: 10.1016/j.jmig.2006.07.008.

10. Le Donne M, Alibrandi A, Ciancimino L, Azzerboni A, Chiofalo B, Triolo O. Endometrial pathology in breast cancer patients: effect of different treatments on ultrasonographic, hysteroscopic and histological findings. Oncol Lett. 2013;5:1305-10. doi: 10.3892/ ol.2013.1156.

11. Gao WL, Zhang LP, Feng LM. Comparative study of transvaginal ultrasonographic and diagnostic hysteroscopic findings in postmenopausal breast cancer patients treated with tamoxifen. Chin Med J (Engl). 2011;124:2335-9.

12. McCluggage WG. My approach to the interpretation of endometrial biopsies and curettings. J Clin Pathol. 2006;59:801-12. doi: 10.1136/jcp.2005.029702.

13. Malpica A. How to approach the many faces of endometrioid carcinoma. Mod Pathol. 2016;29(Suppl 1):S29-44. doi: 10.1038/modpathol.2015.142.
14. Howitt BE, Quade BJ, Nucci MR. Atypical uterine polyps sub-diagnostic of mullerian adenosarcoma: a clinicopathologic analysis of 28 cases with long term followup. Laboratory Investigation. 2012;92:276A7A.

15. Tai LH, Tavassoli FA. Endometrial polyps with atypical (bizarre) stromal cells. Am J Surg Pathol. 2002; 26: 505-9.

16. Rodrigues M, Goez E, Larios K, et al. Atypical stromal cells as a diagnostic pitfall in lesions of the lower female genital tract and uterus: a review and presentation of some unusual cases. Patología. 2009; 47:103-7.

17. Taylor E, Gomel V. The uterus and fertility. Fertil Steril. 2008;89:1-16. doi: 10.1016/j.fertnstert.2007.09.069.

18. Sillo-Seidl G. The analysis of the endometrium of 1,000 sterile women. Hormones. 1971;2:70-5.

19. Lieng M, Istre O, Sandvik L, Qvigstad E. Prevalence, 1-year regression rate, and clinical significance of asymptomatic endometrial polyps: cross-sectional study. J Minim Invasive Gynecol. 2009;16:465-71. doi: 10.1016/j.jmig.2009.04.005.

20. Hinckley MD, Milki AA. 1000 office-based hysteroscopies prior to in vitro fertilization: feasibility and findings. JSLS 2004; 8: 103-107.

21. Lee SC, Kaunitz AM, Sanchez-Ramos L, Rhatigan RM. The oncogenic potential of endometrial polyps: a systematic review and meta-analysis. Obstet Gynecol. 2010;116:1197-205. doi: 10.1097/ AOG.0b013e3181f74864.

22. Lass A, Williams G, Abusheikha N, Brinsden P. The effect of endometrial polyps on outcomes of in vitro fertilization (IVF) cycles. J Assist Reprod Genet. 1999;16:410-5.

23. Onalan R, Onalan G, Tonguc E, Ozdener T, Dogan M, Mollamahmutoglu L. Body mass index is an independent risk factor for the development of endometrial polyps in patients undergoing in vitro fertilization. Fertil Steril. 2009;91:1056-60. doi: 10.1016/j.fertnstert.2008.01.002.

24. Nappi L, Indraccolo U, Di Spiezio Sardo A, et al. Are diabetes, hypertension, and obesity independent risk factors for endometrial polyps? J Minim Invasive Gynecol. 2009;16:157-62. doi: 10.1016/j. jmig.2008.11.004.

25. Dal Cin P, Wanschura S, Kazmierczak B, et al. Amplification and expression of the HMGIC gene in a benign endometrial polyp. Genes Chromosomes Cancer. 1998;22:95-9.

26. Dibi RP, Zettler CG, Pessini SA, Ayub AV, de Almeida SB, da Silveira GP. Tamoxifen use and endometrial lesions: hysteroscopic, histological, and immunohistochemical findings in postmenopausal women with breast cancer. Menopause. 2009;16:293300. doi: 10.1097/gme.0b013e31818af10a.

27. Martinez MR, Marazuela MA, Vallejo MR, Bernabeu RA, Medina TP. Metastasis of lobular breast cancer to endometrial polyps with and without the presence of 
vaginal bleeding. Int J Gynaecol Obstet. 2016;134:1012. doi: 10.1016/j.ijgo.2016.01.005.

28. Vicioso L, Ortega MV, Civico V, Lopez-Beltran A. Synchronous metastasis from lobular carcinoma and primary carcinoma of the endometrium in a patient after tamoxifen therapy. Int J Gynecol Pathol. 2013;32: 66-70. doi: 10.1097/PGP.0b013e318257ded2.

29. Hooker AB, Radder CM, van de Wiel B, Geenen MM. Metastasis from breast cancer to an endometrial polyp; treatment options and follow-up. Report of a case and review of the literature. Eur J Gynaecol Oncol. 2011;32:228-30.

30. Tabrizi AD, Vahedi A, Esmaily HA. Malignant endometrial polyps: Report of two cases and review of literatures with emphasize on recent advances. J Res Med Sci. 2011;16(4):574-9.

31. Kafshdooz T, Mohaddes Ardabili SM, Kafshdooze $\mathrm{L}$, et al. Polymorphism of p53 gene codon 72 in endometrial cancer: correlation with tumor grade and histologic type. Asian Pac J Cancer Prev. 2014;15(22):96603-6.

32. Dastranj Tabrizi A, Ghojazadeh M, Thaghizadeh Anvar $\mathrm{H}$, et al. Immunohistochemical profile of leiomyoma with bizarre nuclei; comparision with conventional leiomyoma, smmoth muscle tumors with uncertain malignant potential and leiomyosarcoma. Adv Pharm Bull. 2015;5(suppl 1): 673-7.

Copyright (c) 2016 The Author(s); This is an open-access article distributed under the terms of the Creative Commons Attribution License (http://creativecommons.org/licenses/by/4.0), which permits unrestricted use, distribution, and reproduction in any medium, provided the original work is properly cited. 